\title{
Linx
}

Revue des linguistes de l'université Paris X Nanterre

38 | 1998

L'acquisition de la temporalité en situation bilingue

\section{Temporalité et récit dans l'acquisition du langage en situation bilingue}

Temporality and narrative in child language acquisition in a bilingual setting

\section{Colette Noyau}

\section{(2) OpenEdition \\ 12 Journals}

Édition électronique

URL : http://journals.openedition.org/linx/847

DOI : $10.4000 /$ linx. 847

ISSN : 2118-9692

Éditeur

Presses universitaires de Paris Nanterre

\section{Édition imprimée}

Date de publication : 1 décembre 1998

Pagination : 7-15

ISSN : 0246-8743

\section{Référence électronique}

Colette Noyau, «Temporalité et récit dans l'acquisition du langage en situation bilingue », Linx [En ligne], 38 | 1998, mis en ligne le 25 juin 2012, consulté le 22 septembre 2020. URL : http:// journals.openedition.org/linx/847 ; DOI : https://doi.org/10.4000/linx.847

Ce document a été généré automatiquement le 22 septembre 2020.

Département de Sciences du langage, Université Paris Ouest 


\title{
Temporalité et récit dans l'acquisition du langage en situation bilingue
}

Temporality and narrative in child language acquisition in a bilingual setting

\author{
Colette Noyau
}

1 Le développement des moyens linguistiques de la temporalité chez l'enfant (unilingue) s'est révélé un domaine extrêmement fécond, il a amené la psycholinguistique à mettre en relation développement cognitif et développement linguistique, pour plusieurs composantes des représentations temporelles et de leur mise en mots.

2 Ainsi, les jeunes enfants expriment d'abord l'aspect avant d'exprimer le temps lorsqu'ils utilisent la morphologie temporo-aspectuelle de leur langue, que cette langue soit à dominante temporelle ou aspectuelle (Bronckart 1976, Ferreiro 1973), phénomène mis en relation avec l'atteinte du stade cognitif de la décentration (cf. aussi Sabeau-Jouannet 1977). Plus récemment, Weist $(1986,1989)$ propose un modèle affiné des étapes cognitives de la maîtrise des systèmes temporo-aspectuels, fondé sur les mises en relation entre les intervalles temporels TP (temps de la parole), TS (temps de la situation) et TR (temps de référence), présenté dans Romeo (ici même) et invoqué également pour rendre compte du développement de la temporalité-aspectualité grammaticale en langue étrangère (cf. Schlyter 1990).

D'après Clark (1995), synthétisant les apports des travaux jusqu'en 1990 concernant l'acquisition des langues romanes sur l'émergence de notions temporelles, et sur l'émergence des temps grammaticaux, les distinctions temporelles grammaticales émergent dans la production à partir de $2 ;-3$; mais la maitrise du système temporel prend plusieurs années, au long desquelles les fonctions des schèmes morphologiques évoluent notamment de l'aspectualité vers la temporalité. Par ailleurs, la mise en relation temporelle de deux événements dans un énoncé - et la compréhension de tels énoncés - est tributaire de l'ordre d'énonciation, quels que soient les connecteurs: avant (de / que), après (- / que) utilisés (cf. Clark 1970, 1973) jusqu'à l'atteinte par 
l'enfant du stade cognitif de la réversibilité postulé par Piaget (cf. notamment Piaget 1946, 1965).

4 Il faut évoquer également la fécondité des travaux psycholinguistiques des trois dernières décennies sur le développement de la capacité narrative chez l'enfant, qui ont permis de décrire les différents niveaux cognitifs à coordonner: cognition des événements complexes, ajustement à l'audience et à la situation d'énonciation, conceptualisation et formulation de propositions formant un tout textuel (Fayol 1985), et l'influence de la langue particulière sur la formulation mais aussi la conceptualisation de récits (Berman \& Slobin 1995).

\section{L'acquisition du langage en situation bilingue}

De multiples questions se posent au chercheur qui s'intéresse aux enfants placés en situation plurilingue : d'abord l'enfant bilingue est un enfant : comme tous les enfants, il vit son appropriation du monde par le développement cognitif et langagier, avec des interactions multiples entre développement cognitif et appropriation du langage. D'une part, le développement linguistique est déterminé, rythmé, par le développement cognitif, il faut que l'enfant ait construit les notions que sa langue met en mots ou en formes pour en acquérir les expressions linguistiques. D'autre part, l'enfant s'approprie des formes de la langue de son environnement et bâtit sur leur sens des hypothèses dépendant de l'étape de développement cognitif qu'il a atteinte.

On peut tout dire en toute langue (en y mettant les moyens, et bien sûr de façon plus ou moins directe ou au contraire coûteuse selon les langues). Mais en même temps, on peut admettre, comme le propose Slobin (1995), que chaque langue particulière offre des façons spécifiques de conceptualiser certains aspects du monde de l'expérience, et ce particulièrement pour des notions grammaticalisées. Chaque langue induit certaines façons de "penser pour parler". Que se passe-t-il lorsque les deux langues auxquelles est exposé un enfant structurent notionnellement le monde de façon différente ? C'est là un champ nouveau pour la typologie des langues (cf. Berman \& Slobin 1995).

7 Le développement des deux langues chez le jeune enfant en situation bilingue (qu'il s'agisse de bilinguisme simultané ou consécutif) fait l'objet de nombreuses recherches européennes récentes. En témoignent notamment les recherches longitudinales sur des enfants bilingues (études de cas ou suivi de groupes d'enfants), dont le programme de recherche DUFDE de Hamburg (Meisel, ed. 1990, 1994) et celui de Lund (Schlyter 1993), une floraison de thèses dans les années 90 sur l'acquisition bilingue du langage (FührerNicod 1994, Jisa 1989, Köppe 1995, Lanza 1990, Müller 1993), l'intérêt de la psycholinguistique (Bialystok 1991) et de la sociolinguistique (Romaine 1995) pour l'enfant bilingue, les livres récents fondés maintenant sur une recherche psycholinguistique approfondie concernant les modalités d'éducation linguistique des enfants en famille bilingue (Arnberg 1987, De Houwer 1990, Döpke 1992), les livraisons de la revue AILE (Acquisition / Interaction / Langue étrangère) 6 sur 'L'acquisition bilingue simultanée préscolaire' (Schlyter, ed. 1995), et 10 sur 'L'appropriation des langues en situation de contact' (Muñoz, Nussbaum \& Pujol, eds. 1997).

8 Depuis la célèbre monographie de Ronjat (1913), les discussions ont porté sur la tâche spécifique assignée à l'enfant placé dans un environnement bilingue de séparer les deux langues lors de la construction du langage. 
On peut se demander si l'acquisition de plusieurs langues premières suit pour chacune les étapes de développement décrites chez l'enfant unilingue. L'enfant placé dans un environnement bilingue doit conquérir le langage à travers l'exposition à deux langues. Il a donc plusieurs tâches à accomplir :

- il lui faudra tout d'abord découvrir qu'il est exposé à deux systèmes sémiotiques distincts ;

- il lui faudra donc apprendre à séparer les deux langues dans le traitement linguistique et à structurer deux ensembles de connaissances ;

- il devra se donner des principes pour sélectionner l'une des langues lors de chaque interaction (en fonction de l'interlocuteur, en fonction du thème, en fonction des rapports existants ou à instaurer ... ) ;

- enfin, s'il est dans une communauté bilingue, pratiquant un parler bilingue où les alternances de code (code switching) sont mobilisées à des fins communicatives, il devra découvrir et apprendre à exploiter les fonctionnalités spécifiques des alternances de code dans ce parler bilingue (pour structurer le discours, pour des effets perlocutoires spécifiques, pour évoquer des univers culturels distincts ...). Cette dimension a été analysée de façon suggestive dans les travaux suisses de Lüdi et Py (1986) et de leurs collaborateurs. L'acquisition du bilinguisme (séparation des langues, choix de la langue, alternances de code) constitue-t-elle un problème spécifique dans le développement linguistique ?

Le développement linguistique en situation plurilingue aura-t-il, en conséquence, une structure particulière? Autrement dit, l'enfant acquerra-t-il simultanément deux "langues premières", de la même façon que chez les enfants monolingues pour ces deux langues? ou bien l'acquisition du langage chez l'enfant en environnement bilingue sera-t-elle marquée par cette situation spécifique?

11 Par ailleurs, lorsqu'il y a asymétrie d'exposition de l'enfant aux deux langues, dans quelle mesure a-t-on acquisition de deux langues premières, ou s'agit-il de l'acquisition d'une langue première (la langue dite 'forte'), et l'acquisition de la langue dite 'faible' relève-t-elle plutôt de l'acquisition d'une langue seconde?

Comment se manifestent la dominance d'une langue sur l'autre, et les changements de dominance entraînés par des changements d'environnement linguistique?

Et dans quelle mesure les phénomènes de dominance permettent-ils de penser la structure de l'acquisition comme régie par le développement cognitif général, ou plutôt par la structuration spécifique du domaine linguistique?

Volterra \& Taeschner (1978) ont proposé un modèle allant de l'indifférenciation à la différenciation progressive des langues par l'enfant en trois phases, longtemps considéré comme avéré et aujourd'hui remis en cause. Les travaux longitudinaux réexaminant de plus près les phases très précoces de l'acquisition bilingue suggèrent maintenant que l'enfant en environnement bilingue dès la naissance développe parallèlement dès le départ deux systèmes séparés (et en plus, dans certains cas un système mixte), et qu'il sait très précocément comment s'adapter au parler de l'interlocuteur, monolingue ou mixte (De Houwer 1995).

Par ailleurs, la comparaison des parcours acquisitionnels en langue a et en langue $b$ chez le même enfant permet de renouveler l'approche typologique de l'acquisition (cf. par exemple Krasinsky 1995) et de la construction de récit (Dart 1992). Ainsi, Tracy (1996) montre sur l'analyse de données précoces d'une enfant bilingue allemand/ anglais que les différences structurelles ne perturbent ni ne retardent l'acquisition; que si on s'abstrait des mélanges lexicaux, explicables entre deux langues si proches, on 
constate que les erreurs structurales se trouvent aussi dans l'acquisition monolingue, et que certaines structures, qui sont superposables dans les deux langues dans des contextes limités, servent à résoudre des problèmes grammaticaux à un moment donné (précurseurs de structures plus complexes); enfin que la grammaire du verbe allemand (positions et morphologie) s'acquiert plus vite que celle de l'anglais, plus difficile car la morphologie anglaise est moins saillante et plus ambigüe.

C'est à cet ensemble de questions que tentent de répondre les travaux actuels sur le développement parallèle des deux langues chez le jeune enfant en situation bilingue, parmi lesquels s'inscrivent les contributions ici rassemblées.

17 Toutes ces contributions reprennent ce dossier du point de vue des enfants acquérant le langage dans un environnement bilingue. Elles ont été initialement présentées lors du colloque international de juin 1995 sur l'acquisition de la temporalité et du récit en situation bilingue. ${ }^{1}$ Les processus d'acquisition linguistique y sont étudiés soit dans des études longitudinales monographiques (les études de cas jouent un rôle central dans le domaine du développement linguistique), soit dans des études tranversales sur des groupes d'enfants de tranches d'âge déterminées et placés dans les mêmes environnements d'acquisition. Toutes sont centrées autour d'un domaine d'expérience: la temporalité, et d'un type d'activité discursive: le récit. ${ }^{2}$ Plusieurs d'entre elles abordent la dimension typologique des langues mises en contact.

\section{Récit et temporalité dans l'acquisition du langage}

Le domaine de la temporalité est omniprésent dans les énoncés et dans le discours, et mobilise une gamme étendue de moyens linguistiques. Toute situation référée doit être située dans le temps. Les procès (états, événements et actions) entretiennent entre eux des relations temporelles (avant, après, concomitant, de proximité, d'inclusion ...), ils possèdent des caractéristiques temporelles intrinsèques (ils se caractérisent comme duratifs ou ponctuels, statiques ou évolutifs, débouchant ou non sur un état distinct), ils peuvent être envisagés selon différentes perspectives (cf. Klein 1994). Tous ces ensembles de notions sont catégorisés par les langues particulières de façon diverse, et leur expression met à contribution de façon diverse et avec des poids variables le lexique, la syntaxe, la morphologie, l'organisation des discours. L'enfant doit construire ces catégorisations et découvrir les structures qui les portent à mesure que son développement cognitif et langagier le lui permet.

Quant au récit, c'est un type de texte fondamental pour ce qui concerne la dimension temporelle, puisque procès, intervalles temporels et relations temporelles en forment l'ossature constitutive (cf. Combettes, François, Noyau \& Vet 1996). Et c'est en apprenant à raconter que l'enfant s'essaie à interpréter le monde, à doubler les relations temporelles de relations plus fortes, de causalité, d'intentionnalité, ... et à construire des représentations hiérarchisées d'événements complexes. On peut s'appuyer là sur les apports considérables de la recherche sur l'acquisition unilingue.

La thématique de cette rencontre est à la croisée de plusieurs secteurs de recherche : psycho-linguistique développementale, études du plurilinguisme, sémantique cognitive. Ce triple champ détermine un ensemble trop vaste pour pouvoir le parcourir dans son ensemble. Nous avons donc choisi de le limiter de deux façons : nous mettons l'accent sur l'acquisition du langage et des langues, plus que sur l'acquisition du bilinguisme; et nous nous intéressons à un seul domaine notionnel, central dans le 
langage, et particulièrement fécond car il concerne tous les aspects de la structuration du langage : le domaine du temps. Toutes les contributions ici rassemblées portent sur l'appropriation par l'enfant bilingue des moyens d'exprimer la temporalité dans ses langues - et ce de façon privilégiée à travers l'activité de construction de récits.

Les auteurs ont tenté d'apporter, à partir de situations linguistiques variées, et sous l'angle spécifique de l'expression du temps et des conduites narratives, des éléments de réponse aux grandes questions évoquées ci-dessus.

Ces travaux illustrent des situations bilingues alliant le français à des langues variées, notamment en ce qui concerne la structuration du domaine notionnel de la temporalité et son expression linguistique :

- une langue étroitement apparentée génétiquement et typologiquement (l'italien),

- des langues indo-européennes de famille différente (le français langue romane face à l'allemand ou à l'anglais langues germaniques),

- et des langues éloignées, de caractéristiques typologiques diverses: le coréen, le turc, le mpongwé langue bantoue.

Il est crucial que les études acquisitionnelles, et en particulier celles sur l'acquisition bilingue, s'appuient sur un large éventail translinguistique. C'est en confrontant ce qui se passe pour différents couples de langues que l'on peut éviter un certain linguocentrisme, et éviter les écueils à la fois de la simple collection de faits empiriques, et des généralisations abusives, en tentant de caractériser les processus généraux d'acquisition et de traitement du langage, tels qu'ils sont mis en œuvre sur des matériaux langagiers diversement organisés. Les faits d'acquisition sont à examiner selon une dimension typologique.

Mehmet-Ali AKINCI étudie la mise en œuvre de la morphologie temporelle dans des récits sur images chez des enfants bilingues de familles immigrées turques élevés en France, âgés de 5 à 10 ans, comparés à des enfants turcs monolingues et à des bilingues turc-hollandais aux Pays-Bas. Les enfants étudiés en France tendent à fluctuer entre divers temps dans le même récit, ce qui ne renvoie pas à des alternances narratives de perspective actancielle et aspectuelle comme chez les monolingues, mais plutôt à une approche descriptive simplifiant la tâche narrative (chez les sujets les plus jeunes surtout). Par ailleurs l'évolution vers une maitrise plus mature du système temporoaspectuo-modal du turc est plus lente que chez les enfants monolingues, mais également que chez les bilingues des Pays-Bas, ce qui renvoie d'une part à des habitudes culturelles liées à des environnements socio-culturels plus ou moins favorisés, d'autre part à un facteur éducatif (politique scolaire d'intégration en France vs politique de maintien des langues et cultures d'origine aux Pays-Bas). Ses résultats semblent indiquer que chez ces enfants, le turc, langue première, a le statut de langue faible.

Jin Nam CHOI confronte les récits sur images en français et en coréen d'enfants de familles coréennes en France âgés de sept à huit ans, dont l'expérience du français est surtout liée à la scolarisation. Malgré les différences typologiques importantes entre les deux langues, la structuration du récit se fait dans les deux langues à partir d'une organisation prototypique du récit où les propositions de la trame contiennent des procès transitionnels ('à 2 états', cf. Klein 1994), associés à un temps perfectif, alors que les propositions de l'arrière-plan contiennent des types de procès et des temps verbaux plus variés. Les différences entre les langues apparaissent plus nettes au niveau des connecteurs : les récits coréens et français sont de longueur à peu près égale, mais en 
français, ce sont les propositions de la trame qui sont davantage introduites par des connecteurs (dont $2 / 5$ de 'et'), celles de l'arrière-plan étant le plus souvent sans connecteur, alors qu'en coréen, la grande majorité des propositions, de la trame, et encore davantage de l'arrière-plan, sont introduites par des connecteurs explicitant les relations temporelles et/ou causales. Des comparaisons avec des enfants monolingues français d'une part, coréens d'autre part, devraient permettre de proposer une explication plus fine de ce contraste frappant, qui est bien sûr en relation avec les différences typologiques entre ces deux langues concernant la syntaxe des énoncés complexes.

Marlene DOLITSKY analyse les surgénéralisations translinguistiques effectuées par un enfant bilingue français-anglais pour un marqueur de dérivation lexicale : RE-verbe, et les analyse non comme un transfert d'une langue 'forte' vers la langue la plus 'faible', mais comme un exemple de 'pont' (ou relation privilégiée) tendu par l'enfant entre ses deux langues dans sa tentative de structurer l'ensemble de son environnement linguistique. En s'appuyant sur ce qui est marqué comme 'semblable', l'enfant travaille sur les deux langues en se donnant des règles morphologiques reliant les deux. Vera FÜHRER-NICOD examine le développement syntaxique de la subordination temporelle en allemand et en français chez des enfants bilingues comparés à des enfants germanophones monolingues. Les phases d'acquisition identifiées se retrouvent également chez les enfants monolingues, et les résultats de l'étude semblent indiquer que le transfert de la langue forte vers la langue faible est en ce domaine moindre qu'il n'a été dit antérieurement.

Harriet JISA examine l'évolution du rapport langue forte-langue faible chez une enfant bilingue simultanée anglais-français élevée en France lors de son premier séjour en pays anglophone à l'âge de trois ans. Lors de ce séjour, l'anglais passe très rapidement du statut de langue pour la compréhension, réduite à quelques formules et motsphrases en production, à un statut de langue de communication approchant le niveau de celle des enfants monolingues du même âge. L'évolution des formes verbales et particulièrement le développement du schème de progressif lors de cette période manifeste un parcours de développement très différent de celui des enfants monolingues, la prédominance de l'auxiliaire et le retard des désinences verbales -ing, $e d$, -en s'expliquant par la forte saillance de l'auxiliaire en français oral, qui joue un rôle plus important que les désinences post-verbales pour l'expression du temps et de l'aspect.

Pierrette OGOUAMBA présente quelques aspects des restructurations de la morphologie verbale de la langue mpongwè (langue bantoue) chez 101 enfants de Libreville (Gabon). Le mpongwè est une langue très menacée: tous les enfants sont bilingues, et ont comme langue forte le français. Elle a demandé aux enfants de raconter une histoire à partir d'un livret d'images sans texte. Elle examine à partir de ces corpus les stratégies utilisées par les enfants face au système d'accord sujet-verbe, les emprunts au lexique verbal du français, et la simplification du système temporo-aspectuel.

Silvia ROMEO examine l'acquisition du système de la temporalité chez un enfant bilingue italien-français entre 2 et 4 ans. Cette acquisition est vue comme appropriation graduelle des catégories conceptuelles sous-jacentes aux manifestations linguistiques et inférables grâce à celles-ci. La perspective est développementale; les choix méthodologiques se fondent sur une approche conceptuelle de description des structures temporelles. Les résultats montrent un développement équilibré des deux 
langues, la généralisation de presque tout le paradigme verbal en italien et en français avant $2 ; 4$ avec des formes correspondant aux fonctions de la langue, i. e. plurifonctionnelles. Les perspectives et les relations temporelles ont été acquises simultanément : la théorie dite "du Temps Défectif" est réfutée. En effet dès l'âge de $2 ; 5$ on constate la saisie des notions temporelles caractéristiques du système dit du Temps de Référence. Ces résultats se rapprochent du développement linguistique observé chez les enfants polonais étudiés par Weist plutôt que de celui des enfants italiens ou français. Ces résultats l'amènent à une comparaison typologique entre langues slaves et romanes prenant en compte la formation morphologique du Temps et la sémantique verbale. L'acquisition de deux langues premières, à côté des difficultés acquisitionnelles inhérentes au phénomène du bilinguisme, est vue, dans le cas présenté, comme capable de : faire découvrir des caractéristiques intrinsèques des deux langues, utiles pour la construction du système conceptuel de la temporalité ; aider l'enfant à comprendre la plurifonctionalité des formes; stimuler précocement les opérations cognitives de mémorisation, analyse, comparaison, subdivision, organisation interne. Le type des langues en jeu semble une variable utile.

31 Suzanne SCHLYTER part de l'observation souvent faite que la forme de l'imparfait est difficile à apprendre et souvent remplacée par le présent par les apprenants de français langue étrangère, alors que chez les enfants qui acquièrent le français comme langue maternelle cette forme est correctement utilisée dès le début. Il est montré ici que l'input et la saisie de ces formes diffèrent chez ces deux catégories d'apprenants. Dans la langue adressée aux enfants, les formes d'imparfait se trouvent le plus souvent vers la fin du tour de parole, ce qui facilite la saisie, alors que dans les questions adressées aux apprenants adultes, ces formes se trouvent surtout dans des positions beaucoup moins saillantes, et sont ainsi très rarement saisies. La même étude réalisée auprès d'enfants bilingues indique pourtant que non seulement l'input entre en jeu, mais aussi la propension à saisir la forme: alors que dans leur langue dominante ces enfants semblent saisir très volontiers la forme d'imparfait, cela n'est pas le cas dans la même mesure lorsqu'il s'agit de leur langue faible.

Ricarda SCHNEIDER présente une étude de cas portant sur l'acquisition de la subordination temporelle par un enfant bilingue français-allemand et couvrant la période entre 2 ans 2 mois et 3 ans 4 mois. En partant des structures linguistiques relevées dans les données, l'étude détermine les étapes d'émergence des différentes structures dans les deux langues ainsi que leurs précurseurs et les relations temporelles exprimées (concomitance, antériorité, postériorité). L'objectif de l'analyse était également de préciser dans quels contextes discursifs la subordination temporelle fait ses premières apparitions et se trouve le plus souvent employée (conversation, récit d'expérience personnelle, récit à partir d'images). Les résultats montrent que la subordination temporelle est d'abord employée dans la conversation courante, puis dans les récits d'expériences vécues par l'enfant, pour n'apparaître que plus tardivement dans les récits produits à partir d'images. Son acquisition et son emploi suivent un développement quasi-parallèle dans les deux langues. 


\section{BIBLIOGRAPHIE}

ARNBERG Lenore (1987): Raising children bilingually: the pre-school years. Clevedon (Avon):

Multilingual Matters.

BERMAN, Ruth \& Slobin, Dan (1994), Different Ways of Relating Events in Narrative: A

Crosslinguistic Developmental Study, Hillsdale, NJ, Erlbaum.

BIALYSTOK Ellen (ed.) (1991): Language processing in bilingual children. Cambridge Univ. Press.

BRONCKART, Jean-Paul (1976) : Genèse et organisation des formes verbales chez l'enfant. De l'aspect au temps. Bruxelles, Dessart et Mardaga.

CLARK Eve (1970) : How young children describe events in time. In : FLORES D'ARCAIS Ino \& Pim LEVELT (eds.) Advances in psycholinguistics. Amsterdam, North-Holland, 275-284.

CLARK Eve (1973): How children describe time and order. In : FERGUSON Ch. \& Dan SLOBIN (eds.) Studies in child language development. New York, Holt, Rinehart \& Winston, 585-606.

CLARK Eve (1985): The acquisition of Romance, with special reference to French. In D. SLOBIN ed. The cross-linguistic study of language acquisition vol. I The data. Hillsdale (N.J.), Erlbaum, 687-782.

COMBETTES Bernard, François Jacques, NoyAu Colette \& Co VeT (1993) : Introduction à l'étude des aspects dans le discours narratif. Verbum 4/1993 'Les aspects dans le discours narratif I' , 5-48.

DART Sarah (1992): Narrative style in the two languages of a bilingual child. Journal of Child language 19, 367-387.

DE Houwer Annick (1990): The acquisition of two languages from birth: a case study. Cambridge University Press.

DE HouWER Annick (1995): Bilingual language acquisition. In P. FLETCHER \& B. MacWHINNEY (Eds.), The Handbook of Child Language . Oxford: Blackwell, 219-250.

DöPKE Susan (1993): One parent - one language. An interactional approach. Amsterdam, Benjamins.

FERREIRO Emma (1973) : Les relations temporelles dans le langage de l'enfant. Genève : Droz.

FÜHRER-NICOD Vera (1994) : Recherches sur le bilinguisme franco-allemand chez les jeunes enfants. Presses Universitaires de Reims.

JISA Harriet (1989) : Etudes sur l'acquisition du langage chez les enfants monolingues et bilingues. Thèse de doctorat, Université Lumière-Lyon 2,3 vol.

KLEIN Wolfgang (1994), Time in Language, London, New York, Routledge.

KÖPPE, R. (1997) Sprachentrennung im frühen bilingualen Erstspracherwerb Französisch / Deutsch. Series A: Language development 21, Gunter Narr, Tübingen. (thèse 1995).

KRASINSKI Emily (1995): The development of past marking in a bilingual child and the punctual nonpunctual distinction. First language 15/3, 45, 239-276.

LANZA Elisabeth (1990): Language mixing in infant bilingualism: a sociolinguistic perspective. Thèse de doctorat Université de Georgetown (à paraître , Oxford University Press).

LÜDI, Georges \& Bernard PY (1986) : Etre bilingue. Berne, Peter Lang. 
MEISEL Jürgen, ed. (1990): Two first languages. Early grammatical development in bilingual children. Dordrecht, Foris.

MEISEL Jürgen (1994): Bilingual first language acquisition. French and German grammatical development. Amsterdam, John Benjamins.

MÜLLER Natascha (1993) : Komplexe Sätze. Der Erwerb von COMP und von Wortstellungsmustern bei bilingualen Kindern (Französisch / Deutsch). Gunter Narr Verlag, Tübingen.

PIAGET Jean (1946) : Le développement de la notion de temps chez l'enfant. Paris, P.U.F.

PIAGET Jean (1965) : Psychologie et pédagogie, Paris Denoèl-Gonthier, 'Médiations'.

RomaINE Suzanne (1995): Bilingualism. Oxford, Basil Blackwell. Chap. 5 : The bilingual child.

RonjAT, J. (1913), Le développement du langage observé chez un enfant bilingue, Paris, Champion.

SABEAU-JOUANNET, Emilie (1977), "L'expression de l'organisation spatiale et temporelle, son

évolution chez des enfants de 2 à 5 ans", chapitre IX de François, F., Fançois, D., Sabeau-Jouannet, E. \& Sourdot, M. La syntaxe de l'enfant avant cinq ans, Paris, Larousse.

SCHLYTER Suzanne (1990): The acquisition of French temporal morphemes in adults and in bilingual children. In : G. BERNINI \& A. GIACALONE RAMAT (eds.) La temporalità nell'acquisizione di lingue seconde. Milano, Franco Angeli.

SCHLYTER Suzanne (1993): The weaker language in bilingual Swedish-French children. In: K. HYLTENSTAM \& Å. VIBERG (eds.) Progression and regression in language. Cambridge University Press.

SLOBIN Dan (1995): From “Thought and language” to "thinking for speaking”. In: GUMPERZ J. \& S. LEVINSON, eds. Rethinking linguistic relativity. Cambridge University Press.

TRACY Rosemary (1996) : Vom Ganzen und seinen Teilen : Überlegungen zum doppelten Erstspracherwerb.Sprache und Kognition 15/1-2, 70-92.

VOLTERRA Virginia \& Traute TAESCHNER (1978): The acquisition and the development of language by bilingual children. Journal of Child Language 5: 311-325.

WEIST, R. M. (1986): Tense and aspect. In: P. FLETCHER \& M. GARMAN (eds.) Language acquisition: studies in first language development (2e éd.). Cambridge, Cambridge University Press, 356-374.

WEIST, R. M. (1989): Time concepts in language and thought: filling the Piagetian void from two to five years. Chap. 2 de: LEVI I. \& D. ZAKAY (eds.) Time and human cognition: a life span perspective.Amsterdam, Elsevier (North-Holland), 63-116.

\section{NOTES}

1. A l'exception de la contribution de Choi, issue d'une recherche en cours allant dans le même sens.

2. Outre les auteurs figurant dans ce $\mathrm{n}^{\circ}$, participaient à la rencontre d'autres chercheurs, en tant que rapporteurs des communications présentées et chargés de synthétiser les discussions: Christian Champaud, Françoise Cordier, Maya Hickmann, Marie-Louise Le Rouzo, Emilie SabeauJouannet, ainsi que Jacques Anis et Eliane Koskas en tant que présidents de séance. Nous les remercions tous chaleureusement de leurs apports. 


\section{RÉSUMÉS}

Les contributions ici rassemblées portent sur l'appropriation par l'enfant bilingue des moyens d'exprimer la temporalité dans ses langues - et ce de façon privilégiée à travers l'activité de construction de récits. L'accent est mis sur l'acquisition du langage et des langues, plus que sur l'acquisition du bilinguisme; et les contributions sont centrées sur un domaine notionnel central dans le langage, et particulièrement fécond, car il concerne tous les aspects de sa structuration : le domaine de la temporalité.

On tente d'apporter, à partir de situations linguistiques variées, et sous l'angle spécifique de l'expression du temps et des conduites narratives, des éléments de réponse aux grandes questions suivantes :

-L'acquisition de plusieurs langues premières suit-elle pour chacune les étapes de développement décrites chez l'enfant unilingue?

- Comment se manifestent la dominance d'une langue sur l'autre et le changement de dominance?

- Dans quelle mesure, lorsqu'il existe une asymétrie d'exposition de l'enfant aux deux langues, at-on acquisition simultanée de deux langues premières, ou acquisition successive d'une langue première et d'une langue seconde?

- Dans quelle mesure les phénomènes de dominance montrent-ils que la structure de l'acquisition est régie par le développement cognitif général ou par la structuration spécifique du domaine linguistique?

The contributions of the present volume explore the ways bilingual children acquire means to express temporality in their two languages, especially in narratives. The spotlight is on language acquisition and acquisition of specific languages, rather than on the acquisition of bilingualism. All the studies bear on a conceptual domain which is central in language, and particularly fruitful as an acquisitional problem since it takes all the linguistic levels together: temporality.

From the consideration of varied linguistic situations, envisaged from the viewpoint of expression of time and narrative production, we seek to answer the following questions:

- Does the acquisition of two or more first languages follow the acquisitional steps that have been described for monolingual acquisition for each of them?

- How are language dominance and dominance change manifested?

- When exposure to both languages is assymetrical, does the child acquire two first languages, or one first language and one second language?

- Do language dominance phenomena allow to state how the structure of acquisition is driven by general cognitive development, or by the specific linguistic structure?

\section{AUTEUR}

\section{COLETTE NOYAU}

Département des Sciences du langage, Université de Paris X - Nanterre, cnoyau@u-paris10.fr 